\title{
A Two-Grid Finite-Volume Method for the Schrödinger Equation
}

\author{
Hongmei Zhang ${ }^{1}$, Jianghua Yin ${ }^{2}$ and Jicheng Jin ${ }^{1, *}$ \\ 1 School of Science, Hunan University of Technology, Zhuzhou, Hunan 412007, China \\ 2 Zhuzhou OKE Precision Cutting Tools Co., Ltd., Zhuzhou, Hunan 412008, China
}

Received 13 July 2019; Accepted (in revised version) 26 October 2019

\begin{abstract}
In this paper, some two-grid finite-volume methods are constructed for solving the steady-state Schrödinger equation. The method projects the original coupled problem onto a coarser grid, on which it is less expensive to solve, and then prolongates the approximated coarse solution back to the fine grid, on which it is not much more difficult to solve the decoupled problem. We have shown, both theoretically and numerically, that our schemes are more efficient and achieve asymptotically optimal accuracy as long as the mesh sizes satisfy $h=\mathcal{O}\left(H^{2}\right)$.
\end{abstract}

AMS subject classifications: $65 \mathrm{~N} 50,65 \mathrm{~N} 30$

Key words: Schrödinger equation, coupled equation, finite volume, two-grid.

\section{Introduction}

In this paper, we will study two-grid finite volume element discretization schemes for the following boundary value problem of the steady-state Schrödinger equation [1]:

$$
\begin{array}{ll}
-\Delta \psi(\mathbf{x})+V(\mathbf{x}) \psi(\mathbf{x})=f(\mathbf{x}), & \forall \mathbf{x} \in \Omega, \\
\psi(\mathbf{x})=0, & \forall \mathbf{x} \in \partial \Omega,
\end{array}
$$

where $\Omega \subset R^{2}$ is a convex polygonal domain, $f(\mathbf{x}), V(\mathbf{x})$ and unknown function $\psi(\mathbf{x})$ are complex-valued.

For any complex-valued function $\psi$, we denote its real part by $\psi_{1}$, the imaginary part by $\psi_{2}$. Then problem (1.1a)-(1.1b) is equivalent to the following coupled equations:

$$
\begin{array}{ll}
-\Delta \psi_{1}(\mathbf{x})+V_{1}(\mathbf{x}) \psi_{1}(\mathbf{x})-V_{2}(\mathbf{x}) \psi_{2}(\mathbf{x})=f_{1}(\mathbf{x}), & \forall \mathbf{x} \in \Omega, \\
-\Delta \psi_{2}(\mathbf{x})+V_{1}(\mathbf{x}) \psi_{2}(\mathbf{x})+V_{2}(\mathbf{x}) \psi_{1}(\mathbf{x})=f_{2}(\mathbf{x}), & \forall \mathbf{x} \in \Omega, \\
\psi_{j}(\mathbf{x})=0, \quad j=1,2, & \forall \mathbf{x} \in \partial \Omega .
\end{array}
$$

*Corresponding author.

Emails: 1045562230@qq.com (H. M. Zhang), yjh10180@163.com (J. H. Yin), jcjin2008@sina.com (J. C. Jin) 
Finite volume method has the local conservation of certain physical quantities and the convenience in numerical implementation, so it has been efficiently used in lot of practical computations and extensively studied in theory [2-19, 35-43]. Zhou et al. constructed symmetric finite volume schemes for selfadjoint elliptic problems [2] in 2002 and for parabolic problems [6] in 2003 respectively. Also, there are different finite volume methods for elliptic problems [9,11-13,15]. Wang et al. [7] develop a second-order finite volume scheme to simulate three dimensional truncated pyramidal QDs in 2006, where the scheme has successfully computed all the confined energy states and visualized the corresponding wave functions. He et al. [8] proposed finite volume method based on stabilized finite elements for the nonstationary Navier-Stokes problem in 2007, where the resulting solution, verified by theoretical analysis and numerical experiments, achieves optimal accuracy, and so on.

On the other hand, the two-grid discretization method, proposed originally by $\mathrm{Xu}$ [20] in 1992, is an efficient numerical method. And it was further investigated and applied to solving many problems, such as nonlinear parabolic equations [21], nonlinear elasticity problems [22], nonlinear PDEs [23], Navier-Stokes equations [24, 25], evolution equations [26], two-phase mixed-domain PEMFC model [27], nonlinear natural convection system [28], Schrödinger equations [1,29-34] and so on.

Later on, more authors connected finite volume method with two-grid method and obtained some important results, for instance, Bi et al. [35] constructed two-grid finite volume element method for linear and nonlinear elliptic problems in 2007; Chen et al. proposed semi-discrete two-grid finite volume element method for semilinear parabolic [36] and for second-order nonlinear hyperbolic equations [37] respectively in 2010; For nonlinear parabolic equations, Chen et al. [38] in 2009 and Zhang et al. [39] in 2011 constructed full-discrete two-grid finite volume element method respectively; Also Zhang [40] proposed two-grid characteristic finite volume element method for nonlinear parabolic equations in 2013; And Zhang [41] constructed semi-discrete two-grid finite volume element method for nonlinear convection-diffusion problems in 2011; Chen et al. [42] proposed two-grid characteristic finite volume element method for semilinear advection-dominated diffusion equations in 2013; Li et al. [43] show both wavelet preconditioners and multilevel preconditioners of linear systems which resulted from the finite volume method for elliptic boundary value problems in 2012. In the above results, some rigorous theoretical analyses are given, and some numerical experiments are presented to confirm the theoretical findings.

In this paper, we explore the two-grid finite volume method to decouple the systems of partial differential equations (1.2a)-(1.2c). Specifically, we extended the approach given in $[2,6]$ to solve the original problem directly on the coarse grid, and constructed a new finite volume method to solve the decoupled equations on the fine grid. The resulting solution, verified by theoretical analysis and numerical experiments, achieves optimal accuracy $\left(h+H^{2}\right)$ in $H^{1}$-norm.

The rest of the paper is organized as follows: Section 2 is a description and analysis of the finite volume method for Schrödinger equation. In Section 3, we construct the 
two-grid finite volume schemes and derive the error estimates. In Section 4, we provide numerical examples to verify the efficiency and effectiveness of the schemes.

\section{The finite volume approximation}

For any real-valued and complex-valued function, the inner product $(\cdot, \cdot)$ and standard Sobolev norms $\left(\|\cdot\|_{m, p},\|\cdot\|_{m, \infty}\right)$ have been defined in the same way as in [44].

Then $\psi(\mathbf{x})$, the weak solution of problem (1.2a)-(1.2c), is defined as follows: Find $\psi(\mathbf{x}) \in H_{0}^{1} \times H_{0}^{1}$ such that

$$
A(\psi, \chi)=(f, \chi), \quad \forall \chi \in H_{0}^{1} \times H_{0}^{1},
$$

where

$$
\begin{aligned}
& A(\psi, \chi)=\left(\nabla \psi_{1}, \nabla \chi_{1}\right)+\left(\nabla \psi_{2}, \nabla \chi_{2}\right)+\left(V_{1} \psi_{1}-V_{2} \psi_{2}, \chi_{1}\right)+\left(V_{1} \psi_{2}+V_{2} \psi_{1}, \chi_{2}\right), \\
& (f, \chi)=\left(f_{1}, \chi_{1}\right)+\left(f_{2}, \chi_{2}\right) .
\end{aligned}
$$

Throughout this paper, we assume that

$$
f_{1}(\mathbf{x}), f_{2}(\mathbf{x}) \in L^{2}(\Omega) ; \quad V_{1}(\mathbf{x}), V_{2}(\mathbf{x}) \in L^{\infty}(\Omega) \quad \text { and } \quad V_{2}(\mathbf{x}) \geq 0 .
$$

Under the assumption (2.3), the variational problem (2.1) has a unique solution $\psi_{h} \in$ $H^{2}(\Omega) \times H^{2}(\Omega)$, which satisfies (see, e.g., [1, Theorem 1]),

$$
\|\psi\|_{2} \leq\|f\|
$$

and the bilinear form $A(\cdot, \cdot)$ satisfies

$$
A(\chi, \chi) \gtrsim\|\chi\|_{1}^{2}, \quad \forall \chi \in H_{0}^{1} \times H_{0}^{1} .
$$

Let $T_{h}$ be a quasi-uniform triangulation of $\Omega$ with mesh size $h>0, Z_{h}$ be the set of all nodes or vertices of $T_{h}, S_{0}^{h} \subset H_{0}^{1}$ be the corresponding piecewise linear finite element space.

For simplicity, let the notation " $\lesssim$ " be equivalent to " $\leq C^{\prime \prime}$ and " $\gtrsim$ " be equivalent to " $\geq C$ " for some positive constant $C$.

Then $\psi_{h}(\mathbf{x})$, the finite element approximation of problem (2.1), is defined as the following:

Find $\psi_{h}(\mathbf{x}) \in S_{0}^{h} \times S_{0}^{h}$ such that

$$
A\left(\psi_{h}, \chi_{h}\right)=\left(f, \chi_{h}\right), \quad \forall \chi_{h} \in S_{0}^{h} \times S_{0}^{h} .
$$

It was shown in [1, Theorem 2] that

$$
\left\|\psi-\psi_{h}\right\|_{s} \lesssim h^{2-s}\|\psi\|_{2}, \quad s=0,1
$$

where $\psi_{h}$ satisfies (2.6). 


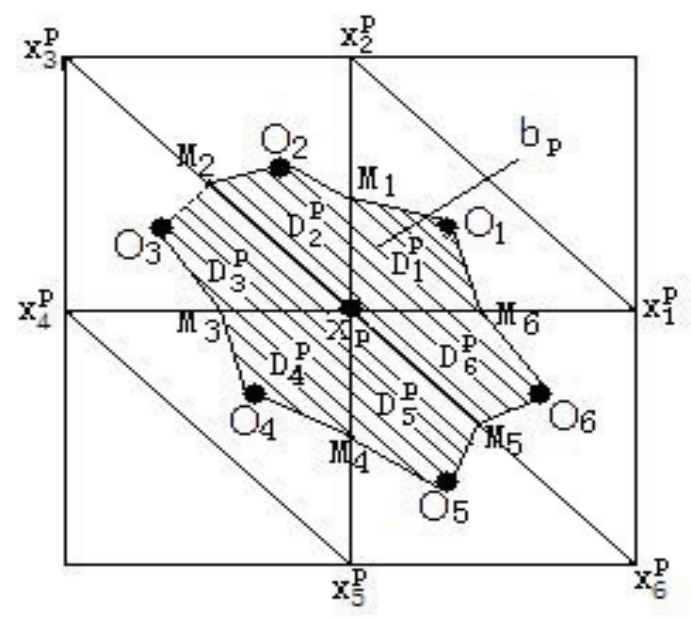

Figure 1: Barycenter Control Volume $b_{p} \in B^{h}$.

Now we construct a barycenter dual mesh $B^{h}$ based upon $T^{h}$ in the same way as in $[2,11,12,35]$, as follows: for each $e \in T^{h}$, find the barycenter point $O_{i}$ of $e$, then connect $O_{i}$ by straight-line segments to the edge midpoints $M_{j}$ of $e$. These segments decompose each $e$ into three subregions. With each vertex $x_{p} \in Z_{h}$, we associate the barycenter control volume $b_{p} \in B^{h}$ (see Fig. 1), which consists of the union of the subregions sharing the vertex $x_{p}$.

Associated with $b_{p}$, we define a constant finite element spaces by

$$
V_{B}=\left\{w \in L^{\infty}(\Omega):\left.w\right|_{b_{p}}=\text { constant }, \forall b_{p} \in B^{h}\right\} .
$$

We then introduce $I_{h}: C(\bar{\Omega}) \rightarrow V_{B}$ satisfying

$$
I_{h} w(x)= \begin{cases}w\left(x_{p}\right), & \forall x \in b_{p} \in B^{h} \\ 0, & \text { others. }\end{cases}
$$

The interpolation operator $I_{h}$ has the following properties $[11,35,39]$.

Lemma 2.1. Let $e \in T^{h}$, For any $\chi \in S_{0}^{h}$, we have

$$
\begin{aligned}
& \int_{e}\left(\chi-I_{h} \chi\right) d x=0 \\
& \left\|\chi-I_{h} \chi\right\|_{0, q, e} \lesssim h_{e}|\chi|_{1, q, e}, \quad 1 \leq q \leq \infty
\end{aligned}
$$

Now we formulate the finite volume method for the problems (1.2a)-(1.2b) as follows. For any vertex $x_{p}$, integrating (1.2a)-(1.2b) over the barycenter control volume $b_{p}$ and 
using Green's formula, we obtain

$$
\begin{cases}-\int_{\partial b_{p}} \frac{\partial \psi_{1}}{\partial n} d s+\int_{b_{p}}\left(V_{1} \psi_{1}-V_{2} \psi_{2}\right) d x=\int_{b_{p}} f_{1} d x, & \forall b_{p} \in B^{h} \\ -\int_{\partial b_{p}} \frac{\partial \psi_{2}}{\partial n} d s+v \int_{b_{p}}\left(V_{2} \psi_{1}+V_{1} \psi_{2}\right) d x=\int_{b_{p}} f_{2} d x, & \forall b_{p} \in B^{h}\end{cases}
$$

where $n$ denotes the unit outer-normal of the domain.

In order to decouple the problems (1.2a)-(1.2b), approximating (2.10), we get the standard finite volume scheme (see, e.g., [2]):

Find $\psi^{h} \in S_{0}^{h} \times S_{0}^{h}$ such that

$$
\left\{\begin{array}{l}
-\int_{\partial b_{p}} \frac{\partial \psi_{1}^{h}}{\partial n} d s+\int_{b_{p}} V_{1} I_{h} \psi_{1}^{h} d x-\int_{b_{p}} V_{2} I_{h} \psi_{2}^{h} d x=\int_{b_{p}} f_{1} d x, \quad \forall b_{p} \in B^{h}, \\
-\int_{\partial b_{p}} \frac{\partial \psi_{2}^{h}}{\partial n} d s+\int_{b_{p}} V_{2} I_{h} \psi_{1}^{h} d x+\int_{b_{p}} V_{1} I_{h} \psi_{2}^{h} d x=\int_{b_{p}} f_{2} d x, \quad \forall b_{p} \in B^{h} .
\end{array}\right.
$$

Namely,

$$
\left\{\begin{array}{l}
-\sum_{x_{p} \in Z_{h}} \int_{\partial b_{p}} \frac{\partial \psi_{1}^{h}}{\partial n} I_{h} \chi_{1} d s+\sum_{x_{p} \in Z_{h}} \int_{b_{p}} V_{1} I_{h} \psi_{1}^{h} I_{h} \chi_{1} d x-\sum_{x_{p} \in Z_{h}} \int_{b_{p}} V_{2} I_{h} \psi_{2}^{h} I_{h} \chi_{1} d x \\
=\sum_{x_{p} \in Z_{h}} \int_{b_{p}} f_{1} I_{h} \chi_{1} d x, \quad \forall \chi_{1} \in S_{0}^{h} \\
-\sum_{x_{p} \in Z_{h}} \int_{\partial b_{p}} \frac{\partial \psi_{2}^{h}}{\partial n} I_{h} \chi_{2} d s+\sum_{x_{p} \in Z_{h}} \int_{b_{p}} V_{2} I_{h} \psi_{1}^{h} I_{h} \chi_{2} d x+\sum_{x_{p} \in Z_{h}} \int_{b_{p}} V_{1} I_{h} \psi_{2}^{h} I_{h} \chi_{2} d x \\
=\sum_{x_{p} \in Z_{h}} \int_{b_{p}} f_{2} I_{h} \chi_{2} d x, \quad \forall \chi_{2} \in S_{0}^{h} .
\end{array}\right.
$$

By the following lemma (cf. [2, Lemma 2.1]),

Lemma 2.2. If matrix $\mathcal{A}=\left(a_{i j}\right)_{2 \times 2}$ satisfies $a_{i j} \in V_{B},(1 \leq i, j \leq 2)$, then

$$
-\sum_{b_{p} \in B^{h}} \int_{\partial b_{p}}(\nabla w)^{T} \mathcal{A} n I_{h} v=\int_{\Omega}(\nabla w)^{T} \mathcal{A} \nabla v, \quad \forall w, v \in S_{0}^{h}
$$

we have

$$
-\sum_{x_{p} \in Z_{h}} \int_{\partial b_{p}} \frac{\partial \psi_{i}^{h}}{\partial n} I_{h} \chi_{i} d s=\left(\nabla \psi_{i}^{h}, \nabla \chi_{i}\right), \quad i=1,2 .
$$

So we get a finite volume scheme as follows: 
Find $\psi^{h} \in S_{0}^{h} \times S_{0}^{h}$ such that

$$
A_{h}\left(\psi^{h}, \chi\right)=(f, \chi)_{h}, \quad \forall \chi \in S_{0}^{h} \times S_{0}^{h},
$$

where

$$
\begin{gathered}
A_{h}(\psi, \chi)=:\left(\nabla \psi_{1}, \nabla \chi_{1}\right)+\left(V_{1} I_{h} \psi_{1}, I_{h} \chi_{1}\right)-\left(V_{2} I_{h} \psi_{2}, I_{h} \chi_{1}\right) \\
+\left(\nabla \psi_{2}, \nabla \chi_{2}\right)+\left(V_{2} I_{h} \psi_{1}, I_{h} \chi_{2}\right)+\left(V_{1} I_{h} \psi_{2}, I_{h} \chi_{2}\right), \\
(f, \chi)_{h}=:\left(f, I_{h} \chi\right)=:\left(f_{1}, I_{h} \chi_{1}\right)+\left(f_{2}, I_{h} \chi_{2}\right) .
\end{gathered}
$$

Next we introduce the norm as follows:

$$
\|w\|_{h}=:\left(I_{h} w, I_{h} w\right)^{\frac{1}{2}}, \quad \forall w \in S_{0}^{h} \times S_{0}^{h} .
$$

So we can get

Lemma 2.3. The norm $\|w\|_{h}$ is equivalent to the norm $\|w\|$, namely

$$
\|w\|_{h} \lesssim\|w\| \lesssim\|w\|_{h}, \quad \forall w \in S_{0}^{h} \times S_{0}^{h} .
$$

Following from (2.16a) and (2.18), we have

Lemma 2.4. Assume that (2.3), then $A_{h}(\cdot, \cdot)$, the bilinear form in (2.16a), satisfies

$$
\begin{array}{ll}
\left|A_{h}(\psi, \chi)\right| \lesssim\|\psi\|_{1}\|\chi\|_{1}, & \forall \psi, \chi \in S_{0}^{h} \times S_{0}^{h}, \\
A_{h}(\psi, \psi) \gtrsim\|\psi\|_{1}^{2}, & \forall \psi \in S_{0}^{h} \times S_{0}^{h} .
\end{array}
$$

Therefore, by the Lax-Milgram theorem and Lemma 2.4, the problems (1.2a)-(1.2b) havs a unique solution $\psi^{h} \in S_{0}^{h} \times S_{0}^{h}$.

Next, to describe error estimates for the finite volume scheme (2.15), we will give some useful lemmas. By Friedrich inequality, we have

Lemma 2.5 ([45]). Let

$$
g_{e}=: \frac{1}{\text { measure }(e)} \int_{e} g d x
$$

if $\forall g \in W^{1, p}(e)$, then

$$
\left\|g-g_{e}\right\|_{0, p, e} \lesssim h\|g\|_{1, p, e}, \quad 1 \leq p \leq \infty
$$

So, we can get

Lemma 2.6. For any $\psi \in S_{0}^{h} \times S_{0}^{h}$, we have

$$
\left|\left(f, \psi-I_{h} \psi\right)\right| \lesssim h^{2}\|f\|_{1, p}\|\psi\|_{1, q,} \quad \forall f \in\left(W^{1, p}(\Omega)\right)^{2},
$$

where $\frac{1}{p}+\frac{1}{q}=1,1 \leq p, q \leq \infty$. 
Proof. Let

$$
f_{i, e}=: \frac{1}{\text { measure }(e)} \int_{e} f_{i} d x, \quad i=1,2, \quad \forall e \in T^{h},
$$

from (2.9a), (2.9b), Lemma 2.5 and Hölder inequality, we obtain

$$
\begin{aligned}
\left|\left(f, \psi-I_{h} \psi\right)\right| & =\left|\sum_{e \in T^{h}} \int_{e}\left[f_{1}\left(\psi_{1}-I_{h} \psi_{1}\right)+f_{2}\left(\psi_{2}-I_{h} \psi_{2}\right)\right] d x\right| \\
& =\left|\sum_{e \in T^{h}} \int_{e}\left[\left(f_{1}-f_{1, e}\right)\left(\psi_{1}-I_{h} \psi_{1}\right)+\left(f_{2}-f_{2, e}\right)\left(\psi_{2}-I_{h} \psi_{2}\right)\right] d x\right| \\
& \lesssim \sum_{e \in T^{h}}\left(\left\|f_{1}-f_{1, e}\right\|_{0, p, e}\left\|\psi_{1}-I_{h} \psi_{1}\right\|_{0, q, e}+\left\|f_{2}-f_{2, e}\right\|_{0, p, e}\left\|\psi_{2}-I_{h} \psi_{2}\right\|_{0, q, e}\right) \\
& \lesssim h \sum_{e \in T^{h}}\left(\left\|f_{1}-f_{1, e}\right\|_{0, p, e}\left|\psi_{1}\right|_{1, q, e}+\left\|f_{2}-f_{2, e}\right\|_{0, p, e}\left|\psi_{2}\right|_{1, q, e}\right) \\
& \lesssim h^{2}\left(\left\|f_{1}\right\|_{1, p}\left|\psi_{1}\right|_{1, q}+\left\|f_{2}\right\|_{1, p}\left|\psi_{2}\right|_{1, q}\right) \\
& \lesssim h^{2}\|f\|_{1, p}\|\psi\|_{1, q} .
\end{aligned}
$$

Thus, we complete the proof.

By the following lemma (cf. (3.10) in [2]),

Lemma 2.7. For any $\alpha \in W^{1, \infty}, w, v \in S_{0}^{h}$, then holds

$$
\left|\left(\alpha I_{h} w, I_{h} v\right)-(\alpha w, v)\right| \lesssim h^{2}\|w\|_{1, p}\|v\|_{1, q}
$$

where $\frac{1}{p}+\frac{1}{q}=1,1 \leq p, q \leq \infty$.

We have

Lemma 2.8. If $V \in\left(W^{1, \infty}\right)^{2}$, then

$$
\left|A(\psi, \chi)-A_{h}(\psi, \chi)\right| \lesssim h^{2}\|\psi\|_{1, p}\|\chi\|_{1, q}, \quad \forall \psi, \chi \in S_{0}^{h} \times S_{0}^{h},
$$

where $\frac{1}{p}+\frac{1}{q}=1,1 \leq p, q \leq \infty$.

Proof. From (2.2a) and (2.16a), noticing that $V \in\left(W^{1, \infty}\right)^{2}$, we have

$$
\begin{aligned}
\left|A(\psi, \chi)-A_{h}(\psi, \chi)\right| \lesssim & \left|\left(V_{1} \psi_{1}, \chi_{1}\right)-\left(V_{1} I_{h} w_{1}, I_{h} \chi_{1}\right)\right|+\left|\left(V_{2} \psi_{2}, \chi_{1}\right)-\left(V_{2} I_{h} \psi_{2}, I_{h} \chi_{1}\right)\right| \\
& +\left|\left(V_{1} \psi_{2}, \chi_{2}\right)-\left(V_{1} I_{h} \psi_{2}, I_{h} \chi_{2}\right)\right|+\left|\left(V_{2} \psi_{1}, \chi_{2}\right)-\left(V_{2} I_{h} \psi_{1}, I_{h} \chi_{2}\right)\right|
\end{aligned}
$$

Therefore, (2.22) follow from (2.21) and (2.23).

Lemma 2.9. $\psi^{h}$, the finite volume solution defined in (2.15), has the estimate:

$$
\left\|\psi^{h}\right\|_{1} \lesssim\|f\|
$$


Proof. From (2.19b), (2.15) and (2.18), we have

$$
\begin{aligned}
&\left\|\psi^{h}\right\|_{1}^{2} \lesssim A_{h}\left(\psi^{h}, \psi^{h}\right)=\left(f, \psi^{h}\right)_{h} \\
& \leq\|f\|\left\|I_{h} \psi^{h}\right\| \lesssim\|f\|\left\|\psi^{h}\right\| .
\end{aligned}
$$

Therefore, (2.24) holds.

Lemma 2.10. If $V \in\left(W^{1, \infty}\right)^{2}, f \in\left(H^{1}\right)^{2}, \psi_{h}$, the finite element solution defined in (2.6), and $\psi^{h}$, the finite volume solution defined in (2.15), have the estimates:

$$
\begin{aligned}
& \left|A\left(\psi^{h}-\psi_{h}, \chi\right)\right| \lesssim h^{2}\|f\|_{1}\|\chi\|_{1}, \quad \forall \chi \in S_{0}^{h} \times S_{0}^{h}, \\
& \left\|\psi_{h}-\psi^{h}\right\|_{1} \lesssim h^{2}\|f\|_{1} .
\end{aligned}
$$

Proof. From (2.6) and (2.15), we have

$$
\begin{aligned}
\left|A\left(\psi^{h}-\psi_{h}, \chi\right)\right| & =\left|A\left(\psi^{h}, \chi\right)-A\left(\psi_{h}, \chi\right)\right|=\left|A\left(\psi^{h}, \chi\right)-(f, \chi)\right| \\
& =\left|A\left(\psi^{h}, \chi\right)-A_{h}\left(\psi^{h}, \chi\right)+(f, \chi)_{h}-(f, \chi)\right| \\
& \lesssim\left|A\left(\psi^{h}, \chi\right)-A_{h}\left(\psi^{h}, \chi\right)\right|+\left|(f, \chi)_{h}-(f, \chi)\right| .
\end{aligned}
$$

Noticing (2.16b), (2.20), (2.22) and (2.24), where $p=q=2$, from (2.26), we have

$$
\begin{aligned}
\left|A\left(\psi^{h}-\psi_{h}, \chi\right)\right| & \lesssim h^{2}\left\|\psi^{h}\right\|_{1, p}\|\chi\|_{1, q}+h^{2}\|f\|_{1, p}\|\chi\|_{1, q} \\
& \lesssim h^{2}\|f\|_{1}\|\chi\|_{1} .
\end{aligned}
$$

Therefore, (2.25a) holds. Furthermore, let $\chi=\psi^{h}-\psi_{h}$ in (2.25a), noticing (4.1), we obtain (2.25b).

Theorem 2.1. Assume that

$$
V \in\left(W^{1, \infty}\right)^{2}, \quad f \in\left(H^{1}\right)^{2} \text { and } \quad V_{1} \geq 0 \quad \text { in } \Omega .
$$

Then $\psi^{h}$, the finite volume approximation of $\psi$, has the error estimate:

$$
\left\|\psi-\psi^{h}\right\|_{s} \lesssim h^{2-s}\|f\|_{1}, \quad s=0,1 .
$$

Proof. From (2.7), (2.25b) and (2.4), we obtain

$$
\begin{aligned}
\left\|\psi-\psi^{h}\right\|_{s} & \leq\left\|\psi-\psi_{h}\right\|_{s}+\left\|\psi_{h}-\psi^{h}\right\|_{1} \lesssim h^{2-s}\|\psi\|_{2}+h^{2}\|f\|_{1} \\
& \lesssim h^{2-s}\left(\|f\|+\|f\|_{1}\right) \lesssim h^{2-s}\|f\|_{1}, \quad s=0,1 .
\end{aligned}
$$

We complete the proof. 


\section{The two-grid finite volume schemes}

In this section, following the idea of decoupling in [1], we construct the two-grid finite volume algorithms for problem (1.1a)-(1.1b). The basic mechanisms in our approach is another finite element space $S_{0}^{H} \times S_{0}^{H}\left(\subset S_{0}^{h} \times S_{0}^{h}\right)$ defined on a coarser quasi-uniform triangulations of $\Omega$ with mesh size $H>h>0$.

Set the bilinear forms

$$
\begin{aligned}
& \hat{A}_{h}(\psi, \chi)=: \int_{\Omega} \nabla \psi_{1} \cdot \nabla \chi_{1} d x+\left(V_{1} I_{h} \psi_{1}, I_{h} \chi_{1}\right)+\int_{\Omega} \nabla \psi_{2} \cdot \nabla \chi_{2} d x+\left(V_{1} I_{h} \psi_{2}, I_{h} \chi_{2}\right), \\
& N_{h}(\psi, \chi)=:\left(V_{2} \psi_{1}, I_{h} \chi_{2}\right)-\left(V_{2} \psi_{2}, I_{h} \chi_{1}\right) .
\end{aligned}
$$

\section{Algorithm 3.1.}

Step 1. Find $\psi^{H} \in S_{0}^{H} \times S_{0}^{H}$ such that

$$
A_{H}\left(\psi^{H}, \chi_{H}\right)=\left(f, \chi_{H}\right), \quad \forall \chi_{H} \in S_{0}^{H} \times S_{0}^{H} .
$$

Step 2. Find $\psi_{v}^{h} \in S_{0}^{h} \times S_{0}^{h}$, such that

$$
\hat{A}_{h}\left(\psi_{v}^{h}, \chi_{h}\right)+N_{H}\left(\psi^{H}, \chi_{h}\right)=\left(f, \chi_{h}\right)_{h}, \quad \forall \chi_{h} \in S_{0}^{h} \times S_{0}^{h} .
$$

Theorem 3.1. If $V \in\left(W^{1, \infty}\right)^{2}, V_{1} \geq 0, f \in\left(H^{1}\right)^{2}$, then $\psi_{v}^{h}$, the two-grid finite volume solution defined in Algorithm 3.1, has the following error estimates:

$$
\left\|\psi^{h}-\psi_{v}^{h}\right\|_{1} \lesssim\left(h+H^{2}\right)\|f\|_{1} .
$$

Consequently,

$$
\left\|\psi-\psi_{v}^{h}\right\|_{1} \lesssim\left(h+H^{2}\right)\|f\|_{1} .
$$

Proof. From (2.15) and (3.2), we have

$$
A_{h}\left(\psi^{h}-\psi_{v}^{h}, \chi\right)=\left(V_{2} I_{h} \psi_{1}^{h}-V_{2} \psi_{1}^{H}, I_{h} \chi_{2}\right)-\left(V_{2} I_{h} \psi_{2}^{h}-V_{2} \psi_{2}^{H}, I_{h} \chi_{1}\right), \quad \forall \chi \in S_{0}^{h} \times S_{0}^{h},
$$

Noticing (2.3), (2.18), (2.9b), (2.24) and (2.28), We obtain

$$
\begin{aligned}
& \left|\left(V_{2} I_{h} \psi_{2}^{h}-V_{2} \psi_{2}^{H}, I_{h} \chi_{1}\right)\right| \\
\leq & \left|\left(V_{2} I_{h} \psi_{2}^{h}-V_{2} \psi_{2}^{h}, I_{h} \chi_{1}\right)\right|+\left|\left(V_{2} \psi_{2}^{h}-V_{2} \psi_{2}^{H}, I_{h} \chi_{1}\right)\right| \\
\leq & \left(\left\|V_{2}\left(I_{h} \psi_{2}^{h}-\psi_{2}^{h}\right)\right\|+\left\|V_{2}\left(\psi_{2}^{h}-\psi_{2}^{H}\right)\right\|\right)\left\|I_{h} \chi\right\| \\
\leq & \left(\left\|I_{h} \psi_{2}^{h}-\psi_{2}^{h}\right\|+\left\|\psi_{2}^{h}-\psi_{2}^{H}\right\|\right)\|\chi\|\left\|V_{2}\right\|_{0, \infty} \\
\lesssim & {\left[h\left|\psi_{2}^{h}\right|_{1}+\left\|\psi_{2}-\psi_{2}^{h}\right\|+\left\|\psi_{2}-\psi_{2}^{H}\right\|\right]\|\chi\|\|V\|_{0, \infty} } \\
\lesssim & {\left[h\|f\|_{0}+\left\|\psi_{2}-\psi_{2}^{h}\right\|+\left\|\psi_{2}-\psi_{2}^{H}\right\|\right]\|\chi\| } \\
\lesssim & \left(h+H^{2}\right)\|f\|_{1}\|\chi\| .
\end{aligned}
$$


Following the idea of (3.5), it holds

$$
\left|\left(V_{2} I_{h} \psi_{1}^{h}-V_{2} \psi_{1}^{H}, I_{h} \chi_{2}\right)\right| \lesssim\left(h+H^{2}\right)\|f\|_{1}\|\chi\| .
$$

We can easily check that

$$
\hat{A}_{h}(w, w) \gtrsim\|w\|_{1}^{2}, \quad \forall w \in S_{0}^{h} \times S_{0}^{h} .
$$

Taking $\chi=\psi^{h}-\psi_{v}^{h}$ in (3.4), from (3.5), (3.6) and (3.7), we obtain

$$
\begin{aligned}
\left\|\psi^{h}-\psi_{v}^{h}\right\|_{1}^{2} & \lesssim\left|A_{h}\left(\psi^{h}-\psi_{v}^{h}, \psi^{h}-\psi_{v}^{h}\right)\right| \\
& \lesssim\left(h+H^{2}\right)\|f\|_{1}\left\|\psi^{h}-\psi_{v}^{h}\right\|,
\end{aligned}
$$

Thus (3.3) holds.

From (2.28) and (3.3), it holds

$$
\begin{aligned}
\left\|\psi-\psi_{v}^{h}\right\|_{1} & \leq\left\|\psi-\psi^{h}\right\|_{1}+\left\|\psi^{h}-\psi_{v}^{h}\right\|_{1} \\
& \lesssim h\|f\|_{1}+\left(h+H^{2}\right)\|f\|_{1} \\
& \lesssim\left(h+H^{2}\right)\|f\|_{1} .
\end{aligned}
$$

So, we complete the proof.

Next, by slightly simplifying the coefficient matrix of the system in Step 2, we can get an alternative of Algorithm 3.2. Let the bilinear forms

$$
\begin{aligned}
& \tilde{A}(\psi, \chi)=: \int_{\Omega} \nabla \psi_{1} \cdot \nabla \chi_{1} d x+\int_{\Omega} \nabla \psi_{2} \cdot \nabla \chi_{2} d x, \\
& \tilde{N}_{h}(\psi, \chi)=:\left(V_{1} \psi_{1}-V_{2} \psi_{2}, I_{h} \chi_{1}\right)+\left(V_{1} \psi_{2}+V_{2} \psi_{1}, I_{h} \chi_{2}\right) .
\end{aligned}
$$

\section{Algorithm 3.2.}

Step 1. Find $\psi^{H} \in S_{0}^{H} \times S_{0}^{H}$ such that

$$
A_{H}\left(\psi^{H}, \chi_{H}\right)=\left(f, \chi_{H}\right), \quad \forall \chi_{H} \in S_{0}^{H} \times S_{0}^{H} .
$$

Step 2. Find $\psi_{v, *}^{h} \in S_{0}^{h} \times S_{0}^{h}$, such that

$$
\tilde{A}_{h}\left(\psi_{v, *}^{h}, \chi_{h}\right)+\tilde{N}_{H}\left(\psi^{H}, \chi_{h}\right)=\left(f, \chi_{h}\right)_{h}, \quad \forall \chi_{h} \in S_{0}^{h} \times S_{0}^{h} .
$$

Theorem 3.2. If $V \in\left(W^{1, \infty}\right)^{2}, V_{1} \geq 0, f \in\left(H^{1}\right)^{2}$, then $\psi_{v, *}^{h}$, the two-grid finite volume solution defined in Algorithm 3.2, has the following error estimates:

$$
\left\|\psi^{h}-\psi_{v, *}^{h}\right\|_{1} \lesssim\left(h+H^{2}\right)\|f\|_{1} .
$$

Consequently,

$$
\left\|\psi-\psi_{v, *}^{h}\right\|_{1} \lesssim\left(h+H^{2}\right)\|f\|_{1}
$$


Proof. From (2.15) and (3.9), we can obtain

$$
\begin{aligned}
\tilde{A}\left(\psi^{h}-\psi_{v, *}^{h} \chi\right)=- & \left(V_{2} I_{h} \psi_{2}^{h}-V_{2} \psi_{2}^{H}, I_{h} \chi_{1}\right)+\left(V_{1} I_{h} \psi_{1}^{h}-V_{1} \psi_{1}^{H}, I_{h} \chi_{1}\right) \\
& +\left(V_{2} I_{h} \psi_{1}^{h}-V_{2} \psi_{1}^{H}, I_{h} \chi_{2}\right)+\left(V_{1} I_{h} \psi_{2}^{h}-V_{1} \psi_{2}^{H}, I_{h} \chi_{2}\right), \quad \forall \chi \in S_{0}^{h} \times S_{0}^{h},
\end{aligned}
$$

we can easily check that

$$
\tilde{A}(w, w) \gtrsim\|w\|_{1}^{2}, \quad \forall w \in S_{0}^{h} \times S_{0}^{h} .
$$

Taking $\chi=\psi^{h}-\psi_{v, *}^{h}$ in (3.12), and right-hand sides following the idea of (3.5), noticing (3.13), we can get

$$
\begin{aligned}
\left\|\psi^{h}-\psi_{v, *}^{h}\right\|_{1}^{2} & \lesssim\left|\tilde{A}\left(\psi^{h}-\psi_{v, *}^{h} \psi^{h}-\psi_{v, *}^{h}\right)\right| \\
& \lesssim\left(h+H^{2}\right)\|f\|_{1}\left\|\psi^{h}-\psi_{v, *}^{h}\right\|,
\end{aligned}
$$

which implies that (3.10) holds. Furthermore, from (2.28) and (3.10), we get

$$
\begin{aligned}
\left\|\psi-\psi_{v, *}^{h}\right\|_{1} & \leq\left\|\psi-\psi^{h}\right\|_{1}+\left\|\psi^{h}-\psi_{v, *}^{h}\right\|_{1} \\
& \lesssim h\|f\|_{1}+\left(h+H^{2}\right)\|f\|_{1} \\
& \lesssim\left(h+H^{2}\right)\|f\|_{1} .
\end{aligned}
$$

Thus, we complete the proof.

\section{Numerical example}

In this section, we carry out the numerical examples to demonstrate the efficiency of our algorithms.

Example 4.1. For problem (1.1a)-(1.1b), let

$$
V=1+i \quad \text { and } \quad V=\frac{1}{2}\left(x_{1}^{2}+x_{2}^{2}\right)+i \frac{1}{2}\left(x_{1}^{2}+x_{2}^{2}\right),
$$

respectively, $\Omega=[0,1] \times[0,1]$, and $f$ be so chosen that

$$
\psi=\left(1-x_{1}\right)\left(1-x_{2}\right) \sin \left(x_{1} x_{2}\right)+i\left(1-x_{1}\right) x_{2} \sin \left[x_{1}\left(1-x_{2}\right)\right]
$$

is the exact solution.

Here $\Omega$ is uniformly divided into families $T_{H}$ and $T_{h}$ of triangulations, and $S_{0}^{H}$, $S_{0}^{h} \subset H_{0}^{1}$ are linear finite element spaces defined on $T_{H}, T_{h}$ respectively. We construct barycenter dual meshes $B^{h}$ and $B^{H}$ based upon $T^{h}$ and $T^{H}$ respectively. For $h=H^{2}$ and $H=1 / 4,1 / 8,1 / 16,1 / 32, \psi_{v}^{h}$ and $\psi_{v, *}^{h}$ are computed by Algorithm 3.1 and Algorithm 3.2, respectively. Also $\psi^{h}$, the standard finite volume solution, and $\psi_{h}^{T}$, two-grid fine element 
Table 1: Errors and CPU time of $\psi_{v}^{h}$ and $\psi^{h}, V=1+i$.

\begin{tabular}{||c|ccc|ccc||}
\hline $\operatorname{mesh}(h)$ & $\left\|\psi-\psi_{v}^{h}\right\|_{1}$ & $r$ & $\mathrm{cpu}(\mathrm{s})$ & $\left\|\psi-\psi^{h}\right\|_{1}$ & $r$ & $\mathrm{cpu}(\mathrm{s})$ \\
\hline $1 / 16$ & $3.247 \mathrm{E}-2$ & & 0.22 & $3.244 \mathrm{E}-2$ & & 0.53 \\
$1 / 64$ & $8.148 \mathrm{E}-3$ & 3.989 & 3.05 & $8.141 \mathrm{E}-3$ & 3.985 & 7.63 \\
$1 / 256$ & $2.038 \mathrm{E}-3$ & 3.998 & 54.37 & $2.036 \mathrm{E}-3$ & 3.998 & 123.72 \\
$1 / 1024$ & $5.094 \mathrm{E}-4$ & 4.000 & 1837.56 & $5.090 \mathrm{E}-4$ & 4.000 & 3331.99 \\
\hline
\end{tabular}

Table 2: Errors and CPU time of $\psi_{v}^{h}$ and $\psi_{h}^{T}, V=\frac{1}{2}\left(x_{1}^{2}+x_{2}^{2}\right)+i \frac{1}{2}\left(x_{1}^{2}+x_{2}^{2}\right)$.

\begin{tabular}{||c|ccc|ccc||}
\hline $\operatorname{mesh}(h)$ & $\left\|\psi-\psi_{v}^{h}\right\|_{1}$ & $r$ & $\mathrm{cpu}(\mathrm{s})$ & $\left\|\psi-\psi_{h}^{T}\right\|_{1}$ & $r$ & $\mathrm{cpu}(\mathrm{s})$ \\
\hline $1 / 16$ & $3.245 \mathrm{E}-2$ & & 0.26 & $3.244 \mathrm{E}-2$ & & 0.29 \\
$1 / 64$ & $8.143 \mathrm{E}-3$ & 3.985 & 4.20 & $8.142 \mathrm{E}-3$ & 3.984 & 0.78 \\
$1 / 256$ & $2.037 \mathrm{E}-3$ & 3.998 & 59.64 & $2.036 \mathrm{E}-3$ & 3.999 & 30.25 \\
$1 / 1024$ & $5.114 \mathrm{E}-4$ & 3.983 & 1463.55 & $5.090 \mathrm{E}-4$ & 4.000 & 3845.05 \\
\hline
\end{tabular}

Table 3: Errors and CPU time of $\psi_{v, *}^{h}$ and $\psi_{h}^{T}, V=1+i$.

\begin{tabular}{||c|ccc|ccc||}
\hline $\operatorname{mesh}(h)$ & $\left\|\psi-\psi_{v, *}^{h}\right\|_{1}$ & $r$ & $\mathrm{cpu}(\mathrm{s})$ & $\left\|\psi-\psi_{h}^{T}\right\|_{1}$ & $r$ & $\mathrm{cpu}(\mathrm{s})$ \\
\hline $1 / 16$ & $3.254 \mathrm{E}-2$ & & 0.21 & $3.253 \mathrm{E}-2$ & & 0.26 \\
$1 / 64$ & $8.159 \mathrm{E}-3$ & 3.988 & 3.84 & $8.162 \mathrm{E}-3$ & 3.986 & 0.74 \\
$1 / 256$ & $2.040 \mathrm{E}-3$ & 4.000 & 55.35 & $2.041 \mathrm{E}-3$ & 3.999 & 30.28 \\
$1 / 1024$ & $5.099 \mathrm{E}-4$ & 4.001 & 1458.30 & $5.102 \mathrm{E}-4$ & 4.000 & 3910.43 \\
\hline
\end{tabular}

Table 4: Errors and CPU time of $\psi_{v, *}^{h}$ and $\psi^{h}, V=\frac{1}{2}\left(x_{1}^{2}+x_{2}^{2}\right)+i \frac{1}{2}\left(x_{1}^{2}+x_{2}^{2}\right)$.

\begin{tabular}{||c|ccc|ccc||}
\hline $\operatorname{mesh}(h)$ & $\left\|\psi-\psi_{v, *}^{h}\right\|_{1}$ & $r$ & $\mathrm{cpu}(\mathrm{s})$ & $\left\|\psi-\psi^{h}\right\|_{1}$ & $r$ & $\mathrm{cpu}(\mathrm{s})$ \\
\hline $1 / 16$ & $3.246 \mathrm{E}-2$ & & 0.17 & $3.244 \mathrm{E}-2$ & & 0.19 \\
$1 / 64$ & $8.144 \mathrm{E}-3$ & 3.986 & 2.73 & $8.141 \mathrm{E}-3$ & 3.985 & 2.84 \\
$1 / 256$ & $2.036 \mathrm{E}-3$ & 4.000 & 50.95 & $2.036 \mathrm{E}-3$ & 4.999 & 64.292 \\
$1 / 1024$ & $5.091 \mathrm{E}-4$ & 3.999 & 1503.67 & $5.090 \mathrm{E}-4$ & 4.000 & 3259.72 \\
\hline
\end{tabular}

solution, are computed by (2.15) and Algorithm A1 in [1] respectively. From the numerical results from Table 1 to Table 4, we can see that

$$
\left\|\psi-\psi_{v}^{h}\right\|_{1} \approx \mathcal{O}\left(H^{2}\right)(\approx \mathcal{O}(h)) \text { and }\left\|\psi-\psi_{v, *}^{h}\right\|_{1} \approx \mathcal{O}\left(H^{2}\right)(\approx \mathcal{O}(h))
$$

which coincide with the theoretical results obtained in Theorem 3.1 and Theorem 3.2, respectively. And, on running CPU time, we find that our two-grid finite volume methods are much more efficient than the standard finite volume method and the two-grid finite element method, when the calculation scale is too high. 


\section{Conclusions}

In this paper, we constructed and analyzed two-grid finite volume schemes for the steady-state Schrödinger equation. The discretization schemes of the coupled equations on a fine grid are reduced to the finite volume schemes of the original equations on a much coarser grid together with the approximated discretization schemes of the decoupled equations on a finer grid. Both theoretical analysis and numerical examples show that our schemes work efficiently and can reach the optimal accuracy in $H^{1}$-norm.

\section{Acknowledgements}

This work is supported by National Science Foundation of China (No. 51973056), Young Scientists Foundation of the Hunan Provincial Science Foundation of China (No. 2017JJ3064), Hunan Provincial Education Department Scientific Research Foundation of China (Nos. 17C0465 and 16C0515).

\section{References}

[1] J. JIN, S. SHU AND J. XU, A two-grid discretization method for decoupling systems of partial differential equations, Math. Comput., 75 (2006), pp. 1617-1626.

[2] S. LIANG, X. MA AND A. ZHOU, A symmetric finite volume scheme for selfadjoint elliptic problems, Int. J. Comput. Appl. Math., 147 (2002), pp. 121-136.

[3] Y. DU, Y. LI AND Z. SHENG, Quadratic finite volume method for a nonlinear elliptic problem, Adv. Appl. Math. Mech., 11 (2019), pp. 838-869.

[4] I. Kissami, M. SEAID AND F. BENKHALDOUn, Numerical assessment of criteria for mesh adaptation in the finite volume solution of shallow water equations, Adv. Appl. Math. Mech., 12 (2020), pp. 503-526.

[5] Y. ZHANG AND M. YANG, A posteriori error analysis of any Order finite volume methods for elliptic problems, Adv. Appl. Math. Mech., 12 (2020), pp. 564-578.

[6] X. MA, S. SHU AND A. ZHOU, Symmetric finite volume discretizations for parabolic problems, Comput. Methods Appl. Mech. Eng., 192 (2003), pp. 4467-4485.

[7] W. WANG, T. HUANG AND H. JIANG, A second-order finite volume scheme for three dimensional truncated pyramidal quantum dot, Comput. Phys. Commun., 174 (2006), pp. 371-385.

[8] G. HE, Y. He AND X. FenG, Finite volume method based on stabilized finite elements for the nonstationary Navier-Stokes problem, Numer. Methods for Partial Differential Equations, 23 (2007), pp. 1167-1191.

[9] P. ChATZIPANTELIDIS, Finite volume methods for elliptic PDE's: a new approach, Math. Model. Numer. Anal., 36 (2002), pp. 307-324.

[10] S. CHOU, D. KWAK AND Q. LI, $L^{p}$ error estimates and superconvergence for covolume or finite volume element methods, Numer. Methods for Partial Differential Equations, 19 (2003), pp. 463-486.

[11] H. WU AND R. LI, Error estimates for finite volume element methods for general second-order elliptic problems, Numer. Methods for Partial Differential Equations, 19 (2003), pp. 593-708. 
[12] P. Chatzipantelidis, V. Ginting AND R. LAZAROV, A finite volume element method for a non-linear elliptic problem, Numer. Linear Algebra Appl., 12 (2005), pp. 515-546.

[13] C. BI, Superconvergence of finite volume element method for a nonlinear elliptic problem, Numer. Methods for Partial Differential Equations, 23 (2007), pp. 220-233.

[14] J. LV AND Y. LI, $L^{2}$ error estimate of the finite volume element methods on quadrilateral meshes, Adv. Comput. Math., 33 (2010), pp. 129-148.

[15] Q. Zou, Hierarchical error estimates for finite volume approximation solution of elliptic equations, Appl. Numer. Math., 60 (2010), pp. 142-153.

[16] Z. CAI, J. MANDEL AND S. MCCORMICK, The finite volume element for diffusion equations on general triangulations, SIAM J. Numer. Anal., 28 (1991), pp. 392-402.

[17] Z. CAI, On the finite volume element method, Numer. Math., 58 (1991), pp. 713-735.

[18] Z. CAI, J. JONES, S. MCCORMICK AND T. RUSSELL, Control-volume mixed finite element methods, Comput. Geosci., 1 (1997), pp. 289-315.

[19] R. EWING, T. LIN, AND Y. LIN, On the accuracy of finite volume element method based on piecewise linear polynomials, SIAM J. Numer. Anal., 39 (2002), pp. 1865-1888.

[20] J. XU, A new class of iterative methods for nonselfadjoint or indefinite problems, SIAM J. Numer. Anal., 29 (1992), pp. 303-319.

[21] Y. CHEN, L. CHEN AND X. ZHANG, Two-grid method for nonlinear parabolic equations by expanded mixed finite element methods, Numer. Methods Partial Differential Equations, 29 (2013), pp. $1238-1256$.

[22] O. AXELSSON AND A. PADIY, On a two level Newton type procedure applied for solving nonlinear elasticity problems, Int. J. Numer. Meth. Eng., 49 (2000), pp. 1479-1493.

[23] O. AXELSSON, W. LAYTON, A two-level discretization of nonlinear boundary value problems, SIAMJ. Numer. Anal., 33 (1996), pp. 2359-2374.

[24] W. Layton And W. Lenferink, Two-level Picard and modified Picard methods for the NavierStokes equations, Appl. Math. Comput., 69 (1995), pp. 263-274.

[25] T. UTNES, Two-grid finite element formulations of the incompressible Navier-Stokes equations, Commun. Numer. Meth. Eng., 13 (1997), pp. 675-684.

[26] M. MARION, AND J. XU, Error estimates on a new nonlinear Galerkin method based on two-grid finite elements, SIAM J. Numer. Anal., 32 (1995), pp. 1170-1184.

[27] M. HE, Z. HUANG, C. WANG AND P. SUN, An efficient two-grid method for a two-phase mixeddomain model of polymer exchange membrane fuel cell, Procedia Computer Science, 9 (2012), pp. 689-698.

[28] Y. YANG, Y. JIANG AND Q. KONG, Two-grid stabilized FEMs based on newton type linearization for the steady-state natural convection problem, Adv. Appl. Math. Mech., 12 (2020), pp. 407-435.

[29] L. IGNAT AND E. ZUAZUA, A two-grid approximation scheme for nonlinear Schrödinger equations: dispersive properties and convergence, C. R. Acad. Sci. Paris, Ser. I, 341 (2005), pp. 381-386.

[30] C. ChIEN, H. HuAnG, B. JENG AND Z. LID, Two-grid discretization schemes for nonlinear Schröinger equations, J. Comput. Appl. Math., 214 (2008), pp. 549-571.

[31] L. WU, Two-grid mixed finite-element methods for nonlinear Schrödinger equations, Numer. Methods Partial Differential Equations, 28 (2012), pp. 63-73.

[32] L. WU, Two-grid strategy for unsteady state nonlinear Schrödinger equations, Int. J. Pure Appl. Math., 68 (2011), pp. 465-475.

[33] H. ZHANG, J. JIN AND J. WANG, Two-grid finite-element method for the two-dimensional timedependent Schrödinger equation, Adv. Appl. Math. Mech., 5 (2013), pp. 180-193.

[34] Z. TIAN, Y. CHEN, Y. HUANG AND J. WANG, Two-grid method for the two-dimensional timedependent Schrödinger equation by the finite element method, Comput. Math. Appl., 77 (2019), 
pp. 3043-3053.

[35] C. BI AND V. GINTING, Two-grid finite volume element method for linear and nonlinear elliptic problems, Numer. Math., 108 (2007), pp. 177-198.

[36] C. CHEN AND W. LIU, Two-grid finite volume element method for semilinear parabolic problems, Numer. Math., 60 (2010), pp. 10-18.

[37] C. CHEN AND W. LIU, A two-grid method for finite volume element approximations of second-order nonlinear hyperbolic equations, J. Comput. Appl. Math., 233 (2010), pp. 2975-2984.

[38] C. CHEN, M. YANG AND C. BI, Two-grid methods for finite volume element approximations of nonlinear parabolic equations, J. Comput. Appl. Math., 228 (2009), pp. 123-132.

[39] T. ZHANG, H. ZHONG AND J. ZHAO, A full discrete two-grid finite-volume method for a nonlinear parabolic problem, Int. J. Comput. Math., 88 (2011), pp. 1644-1663.

[40] T. ZHANG, Two-grid characteristic finite volume methods for nonlinear parabolic problems, J. Comput. Math., 5 (2013), pp. 470-487.

[41] T. ZHANG, The semidiscrete (two-grid) finite volume element method for nonlinear convectiondiffusion problem, Appl. Math. Comput., 217 (2011), pp. 7546-7556.

[42] C. CHEN, W. LIU AND C. BI, A two-grid characteristic finite volume element method for semilinear advection-dominated diffusion equations, Numer. Methods Partial Differential Equations, 29 (2013), pp. 1543-1562.

[43] Y. LI, S. SHU, Y. XU AND Q. ZOU, Multilevel preconditioning for the finite volume method, Math. Comput., 81 (2012), pp. 1399-1428.

[44] J. JIN, N. WEI AND H. ZHANG, A two-grid finite-element method for the nonlinear Schrödinger equation, J. C. M., 33 (2015), pp. 146-157.

[45] S. BRENNER AND L. SCOTT, The Mathematical Theory of Finite Element Methods, New York: Springer, Heidelberg, (1994), pp. 296-302. 